\title{
Utilizing a mixed model approach to compare the lactating rat transcriptome against age-matched control virgins Antony T Athippozhy*1, Liping Huang ${ }^{2}$, Tianyong Zhao', Clavia Ruth Wooton-Kee ${ }^{1}$, Paiboon Jungsuwadee ${ }^{1}$, Arnold J Stromberg ${ }^{2}$ and Mary Vore ${ }^{1}$
}

Address: ${ }^{1}$ Department of Toxicology, University of Kentucky, Lexington, KY 40536, USA and ${ }^{2}$ Department of Statistics, University of Kentucky, Lexington, KY 40506, USA

Email: Antony T Athippozhy* - Antony.Athippozhy@uky.edu

* Corresponding author

from UT-ORNL-KBRIN Bioinformatics Summit 2009

Pikeville, TN, USA. 20-22 March 2009

Published: 25 June 2009

BMC Bioinformatics 2009, I0(Suppl 7):A16 doi:10.1186/147I-2105-10-S7-A16

This abstract is available from: http://www.biomedcentral.com/I47I-2/05/I0/S7/AI6

C 2009 Athippozhy et al; licensee BioMed Central Ltd.

\section{Background}

Lactation requires increased nutrient uptake, as the mother must provide enough nutrition for her offspring while still meeting her own nutritional needs. The lactating dam increases her food intake two-fold to adjust for a three-fold to four-fold increase in energy demands. We have used the Affymetrix Rat Exon 1.0 ST microarray in order to map changes in the liver and small intestine in lactating dams compared against age matched controls.

RNA was extracted from the liver, duodenum, jejunum, and ileum from sixteen rats in each group, and pooled RNA from four rats within the same group was used to create one sample. After removing poorly expressed and unannotated genes, a two factorial mixed model was used to analyze the gene expression level summary data using JMP Genomics $3.2^{\oplus}$. Rat was treated as a random effect, while tissue (liver, duodenum, jejunum, and ileum) and treatment were treated as fixed effects. After obtaining slice p-values for each tissue to judge the effect of lactation within each tissue, a cutoff value of $p<.01$ was established. Ingenuity Pathways ${ }^{\circledast}$ was used to perform a Fisher's exact test to detect categories that are significantly altered based on changes in mRNA levels within each tissue. One of the strongest trends detected was that mRNA from cholesterol synthetic genes increases in at least three of the four tissues with the jejunum showing the least change.
The increase in mRNA of cholesterol synthetic genes is consistent with findings that cholesterol synthesis increases in these tissues $[1,2]$. Further investigation showed that SREBP (Sterol Response Element Binding Protein) stimulated expression of some genes, including those not directly involved in cholesterol synthesis.

\section{Acknowledgements}

Supported by DK46923 and T32ES07266 (ATA; CRW-K)

\section{References}

I. Feingold KR, Moser AH: Effect of lactation on cholesterol synthesis in rats. Am J Physiol 1985, 249:G203-208.

2. Feingold KR, Zsigmond G, Lear SR, Moser AH: Effect of food intake on cholesterol synthesis in rats. Am J Physiol 1986, 25I:G362-G396. 